OPEN ACCESS

Edited by:

Yidong Yang,

University of Science and Technology

of China, China

Reviewed by:

Dong Qian,

University of Science and Technology

of China, China

Zachary D. Horne,

Allegheny Health Network,

United States

*Correspondence:

Jin Wang

wangjin@zjcc.org.cn

Ming Chen

chenming@zjcc.org.cn

${ }^{\dagger}$ These authors have contributed equally to this work

Specialty section:

This article was submitted to

Radiation Oncology,

a section of the journal

Frontiers in Oncology

Received: 21 July 2020 Accepted: 19 October 2020 Published: 10 November 2020

Citation:

Dong B, Zhu X, Shu Z, Ji Y, Lu F, Wang J and Chen M (2020) VideoAssisted Thoracoscopic Lobectomy

Versus Stereotactic Body

Radiotherapy Treatment for EarlyStage Non-Small Cell Lung Cancer: A Propensity Score-Matching Analysis.

Front. Oncol. 10:585709.

doi: 10.3389/fonc.2020.585709

\section{Video-Assisted Thoracoscopic Lobectomy Versus Stereotactic Body Radiotherapy Treatment for Early- Stage Non-Small Cell Lung Cancer: A Propensity Score-Matching Analysis}

\author{
Baiqiang Dong ${ }^{1,2 \dagger}$, Xuan $\mathrm{Zhu}^{3 \dagger}$, Zekai Shu ${ }^{1,2 \dagger}$, Yongling $\mathrm{Ji}^{1,2}$, Fangxiao $\mathrm{Lu}^{4}$, Jin Wang ${ }^{1,2 *}$ \\ and Ming Chen ${ }^{1,2 *}$
}

\begin{abstract}
${ }^{1}$ Department of Radiation Oncology, Cancer Hospital of the University of Chinese Academy of Sciences (Zhejiang Cancer Hospital), Institute of Cancer and Basic Medicine (ICBM), Chinese Academy of Sciences, Hangzhou, China, ${ }^{2}$ Department of Radiation Oncology, Zhejiang Key Laboratory of Radiobiology, Hangzhou, China, ${ }^{3}$ Department of Radiation Oncology, The First Affiliated Hospital, College of Medicine, Zhejiang University, Hangzhou, China, ${ }^{4}$ Department of X-ray, Cancer Hospital of the University of Chinese Academy of Sciences (Zhejiang Cancer Hospital), Institute of Cancer and Basic Medicine (ICBM), Chinese Academy of Sciences, Hangzhou, China
\end{abstract}

Background: Compared the overall outcomes of video-assisted thoracoscopic surgery (VATS) versus stereotactic body radiotherapy (SBRT) for stage I-II non-small cell lung cancer (NSCLC).

Methods: We retrospectively compared overall survival (OS), cancer-specific survival (CSS), locoregional control (LRC), and disease-free survival (DFS) at our institution between January 2012 and December 2016. Propensity score-matching was performed to reduce patient selection bias based on age, gender, Karnofsky performance score, Charlson comorbidity index, pulmonary function, and tumor diameter.

Results: A total of 567 patients treated with SBRT $(n=109)$ or surgery $(n=458)$ were included. Of those, 104 patients were matched for further analyses. Median follow-up was 44 months. At 3 and 5 years, OS was 88.6 and $79.9 \%$ for SBRT, and 94.2 and $91.6 \%$ for surgery $(p=0.097)$. There were no differences noted in 5-year CSS $(83.7$ vs. $91.6 \%$, respectively; $p=0.270$ ). The cumulative incidence of $L R C$ at 3 and 5 years was comparable (93.5 and $93.5 \%$ vs. 94.0 and $85.9 \%$, respectively; $p=0.621$ ). Differences in the rates of disease-free survival at 5 years were not statistically significant (79.0 and $80.5 \%$, respectively; $p=0.624$ ).

Conclusions: This propensity score-matching analysis suggests that SBRT can be an alternative option to VATS lobectomy for stage I-II NSCLC.

Keywords: early-stage non-small cell lung cancer, video-assisted thoracoscopic surgery lobectomy, stereotactic body radiotherapy, propensity score matching, treatment outcome, adverse event 


\section{INTRODUCTION}

The early-stage lung cancer is an increasingly diagnosed disease owing to the widespread use of low-dose computed tomography (CT) screening into routine care $(1,2)$. Lobectomy offers the best potential cure for operable patients with earlystage non-small cell lung cancer (NSCLC) $(3,4)$. However, numerous patients cannot withstand thoracotomy due to comorbidities or personal preference. The minimally invasive video-assisted thoracoscopic surgery (VATS) has been associated with lower complication and faster functional recovery compared with open lobectomy; hence, this approach has gained increasing attention in the previous decades (5-8). From a technological perspective, thoracoscopic lobectomy represents a paradigm shift in surgery. By reducing the surgery-related physiologic insult, minimally invasive surgery expands the pool of operable patients who were previously considered potentially inoperable (9).

Stereotactic body radiotherapy (SBRT), also termed stereotactic ablative radiotherapy, delivers high doses of radiation to restricted volumes over a limited number of fractions. Owing to the steep dose gradients, this approach allows for effective tumor ablation with preservation of the surrounding tissue (10). Both the National Comprehensive Cancer Network Clinical Practice Guidelines and European Society for Medical Oncology Consensus recommend SBRT as a non-surgical treatment option for stage I-II NSCLC $(11,12)$. Additionally, the introduction and continuous advancement of this approach promise to improve outcomes in potentially operable patients (13-17). Recently, in patients with stage I NSCLC, both the use of VATS and SBRT increased (9), therefore, it would be clinical important in decision making for early stage NSCLC patients, especially for those who might tolerate surgery, but at certain risk of surgery. However, no randomized trials comparing minimally invasive lobectomy versus SBRT have been completed by now and retrospective comparisons may be precluded by imbalances in baseline characteristics between both cohorts.

In this study, we performed a propensity score-matching (PSM) analysis to compare the outcomes of both treatments in patients with T1-2N0M0 NSCLC. Our results suggest that both approaches provide similar outcomes, which could provide some clarity to the appropriateness of SBRT and help design and support future randomized trials.

\section{MATERIAL AND METHODS}

\section{Study Population}

All patients undergoing SBRT or VATS lobectomy at Cancer Hospital of the University of Chinese Academy (Hangzhou, China) for T1-2N0M0 clinically confirmed lung cancer from 2012 to 2016 were evaluated. Clinically confirmed lung carcinoma was defined as a primary suspicious mass, partsolid, or ground-glass opacity nodule with speculated or smooth edges shown on CT images that persisted for $\geq 3$ months and showed an increase in its longest axis. In patients in whom radiological results were equivocally correlated, endobronchial ultrasonography or mediastinoscopy was performed at the physician's discretion. Additionally, all patients underwent staging bone scan and brain magnetic resonance imaging. A positron emission tomography/CT (PET/CT) was recommended for all patients and was deemed necessary for diagnosis when biopsy was not considered medically safe or the patient refused to undergo the procedure. All the radiology reviews were blinded to treatment type. Patients with a tumor diameter $>5.0 \mathrm{~cm}$ or those with a biological effective dose (BED) $<100$ Gy were excluded. All disease staging was performed using the Union for International Cancer Control Tumor, Node, Metastasis system ( $7^{\text {th }}$ Edition). The indications were fully examined and discussed among thoracic surgeons and radiation oncologists, all multidisciplinary consultations were well documented.

\section{Treatment Procedures}

Most patients with adequate pulmonary function (forced expiratory volume in $1 \mathrm{~s}$ and diffusing capacity greater than $35 \%$ predicted), arterial oxygen tension greater than $60 \mathrm{mmHg}$, arterial carbon dioxide tension less than $50 \mathrm{mmHg}$, and absence of other contraindicating medical comorbidities, according to the thoracic surgeon, were selected for VATS lobectomy. The operation was performed under general anesthesia with singlelung ventilation through a double lumen tracheobronchial tube on a lateral decubitus position, with a $<5 \mathrm{~cm}$ access incision and full dissection and individual division of hilar structures. Mediastinal lymph node dissection was routinely performed for surgical staging. There were no conversions to open surgery.

Inoperable patients, according to the thoracic surgeon, and those who refused surgical resection were selected for SBRT. The whole course of SBRT was reported in our previous study (1820). The gross tumor volume (GTV) included only the primary tumor; the internal target volume (ITV) was determined using $\mathrm{CT}$ with a four-dimension CT technique, and the tumor motion was assessed. The planning target volume (PTV) was defined as the ITV expanded by a $5-\mathrm{mm}$ margin in each direction. The treatment plans were optimized to limit the administration of high doses to regions of organs at risk. The conformality and dose limits of normal tissues were set according to RTOG0236 (21). In the treatment, $80 \%$ iso-dose line was used as the prescribed dose to cover 95\% PTV, and 100\% iso-dose line was used to cover $100 \%$ ITV. Daily online cone beam CT-based volumetric image-guided radiotherapy using soft tissue target registration was applied prior to all SBRT sessions. The BED was calculated using $\mathrm{BED}_{\alpha / \beta}=$ nd $(1+d / \alpha / \beta)$, where $n=$ number of fractions, $d=$ dose per fraction, and $\alpha / \beta=10 \mathrm{~Gy}$ for the tumor.

\section{Data Collection}

Demographic variables obtained from the electronic file database of Cancer Hospital of the University of Chinese Academy (Hangzhou, China) included age, gender, forced expiratory volume in $1 \mathrm{~s}$ to forced vital capacity ratio (FEV1/FVC\%) and FEV1\% predicted prior to treatment, Karnofsky Performance Status score (KPS), and Charlson comorbidity index (CCI). 
Tumor characteristics included diameter and histology. Complications in the surgery group and toxicity in the SBRT group were scored according to the Common Terminology Criteria for Adverse Events Version 4.0.

Post-treatment follow-up generally consisted of a CT scan of the thorax and upper abdomen performed within 2 months of treatment completion for the first and every 3 months for the first 2 years, and every 4-10 months thereafter. Primary tumor recurrence was diagnosed through histologic confirmation or enlargement of the local tumor on CT that continued for $\geq 6$ months. A PET/CT was considered in case of high suspicion of recurrence. Notably, it is difficult to distinguish between pulmonary fibrosis and tumor recurrence, and intrapulmonary metastasis and secondary primary lung cancer. Therefore, a senior radiologist reviewed post-SBRT imaging to score patterns of failure. Local failure was defined as progression in the same lobe after SBRT or the bronchial stump or port site after surgery. Regional failure was defined as failure in ipsilateral hilar or mediastinal lymph nodes after either treatment. Distant failure indicated recurrence beyond locoregional failure.

The primary endpoint of the study was locoregional control (LRC) and cancer-specific survival (CSS). The latter was defined as the period from the date of treatment to the date of death due to lung cancer or treatment-related mortality. The analysis also focused on disease-free survival (DFS), overall survival (OS), and treatment-related toxicity. DFS was defined as the period from date of surgery or SBRT to the date of any failure, development of a new primary NSCLC, or date of death, with patients censored at the date of the last follow-up. OS was defined as the interval from the date of treatment to any death or the last follow-up.

\section{Statistical Analysis}

The two-tailed $t$ test was used for continuous variables. For nonnormally distributed data, we used the Mann-Whitney $U$ test for comparison. The $\chi^{2}$ test was used for categorical variables. The Kaplan-Meier method was used to calculate the survival rates. PSM was performed using the $\mathrm{R}$ MatchIt package for Windows version. A two-tailed value of $p<0.05$ denoted statistical significance.

\section{RESULTS}

\section{Patient Characteristics}

A total of 567 patients treated with SBRT $(n=109)$ or surgery $(\mathrm{n}=458)$ for stage I-II NSCLC were selected for matching. The exclusions and distribution between treatment methods in the included patients are shown in Figure 1. The baseline characteristics prior to PSM are summarized in Table $\mathbf{1 .}$ Patients who received SBRT exhibited significantly poorer respiratory function, higher CCI, and were older than those who underwent VATS lobectomy. Male patients preferred noninvasive therapy. Seventy-two SBRT patients (66\%), including those without pathological confirmation, underwent PET/CT examination. The prescribed dose mainly delivered $50 \mathrm{~Gy}$ in five fractions $(\mathrm{n}=74,68 \%)$ or 50 Gy in four fractions $(\mathrm{n}=24$,
$22 \%)$. Six patients (5\%) received $60 \mathrm{~Gy}$ in eight fractions, four patients (4\%) received $70 \mathrm{~Gy}$ in ten fractions, and one received 60 Gy in six fractions to the isocenter. All patients received a minimum $\mathrm{BED}_{10}$ of $100 \mathrm{~Gy}$ (range: 100-120 Gy). In the surgery group, all had a complete (R0) resection with negative microscopic margins on final pathologic examination. The number of dissected lymph nodes was $13.3 \pm 6.3$ (mean \pm standard deviation), with $91 \%$ of patients having six or more nodes dissected.

The matching process resulted in a final cohort of 104 patients (52 SBRT and 52 VATS patients) eligible for further analysis. The SBRT and VATS cohorts were similar in terms of age (median: 68 vs. 67 years, respectively), gender, tumor size (median: 2.0 vs. $2.0 \mathrm{~cm}$, respectively), CCI, and respiratory function (Table 2).

\section{Survival}

Follow-up data were complete until May 2019. The median follow-up was 44 months. Seven (14\%), and four (8\%) patients in the SBRT and VATS groups, respectively, died during the follow-up period. Death was tumor-related or attributed to other causes in two (18\%) and nine (82\%) patients, respectively. Other causes included one case of pneumonia and one death of unknown cause. OS was comparable between the two cohorts. The post-SBRT rates at 3 and 5 years were 88.6 and $79.9 \%$, respectively, versus 94.2 and $91.6 \%$ for post-VATS $(p=0.097)$ (Figure 2A). There were no differences noted between SBRT and VATS for the 3- and 5-year CSS (92.9 and 83.7 vs. 94.2 and 91.6\%, respectively; $p=0.270$ ) (Figure $2 B$ ).

Locoregional failure occurred in five and two patients in the SBRT and surgery cohorts, respectively. The rates of LRC did not differ significantly between the groups $(p=0.621)$. The 3 - and 5 year rates of LRC for radiotherapy and surgery were 93.5 and 93.5\% versus 94.0 and $85.9 \%$, respectively (Figure 2C).

Distal failures were observed in 13 patients, (i.e., six and seven patients in the SBRT and surgery groups, respectively). The majority of those $(n=9)$ were intrapulmonary metastases. The median time to any recurrence in patients treated with SBRT or VATS lobectomy was 24.9 and 27.9 months, respectively. DFS at 3 and 5 years was 90.2 and $80.5 \%$ for SBRT versus 79.0 and $84.3 \%$ for surgery $(p=0.624)$ (Figure 2D).

\section{Treatment Toxicity}

Table 3 outlines complications occurring after SBRT and VATS. Adverse events occurring within 6 weeks from SBRT were observed in eight patients (15\%). The most commonly reported complication was increased dyspnea. One patient suffered grade 3 radiation pneumonitis. There was no grade $4-$ 5 toxicity observed in the SBRT group. Systemic reactions were mainly fatigue, anorexia, and dyspnea during treatment; most of these resolved after symptomatic treatment. There was no mortality reported among SBRT patients in the 30-day period after treatment. In the surgery group, 29 patients suffered from complications (56\%), and grade 1-2 complications were observed in 24 patients (46\%). In the VATS group, the mortality rate during the 30 -day period after resection was $2 \%$, 


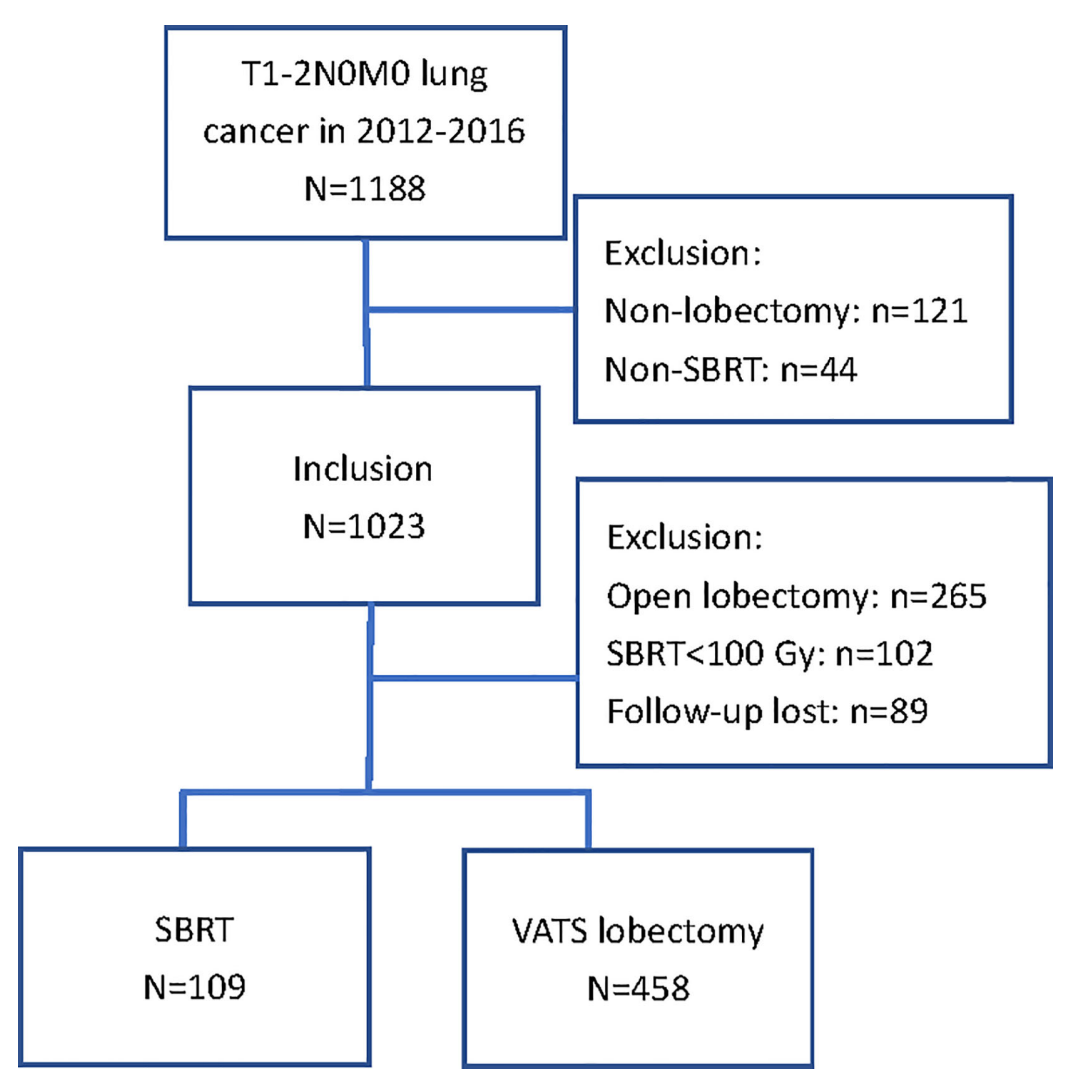

FIGURE 1 | Description of study population; inclusions and exclusions.

with one patient expiring due to multi-organ failure caused by septicemia (consequence of a severe lung infection). Five patients (10\%) experienced grade $\geq 3$ toxicity in the surgery group. One and two patients treated with SBRT and VATS lobectomy, respectively, required rehospitalization within 90 days.

\section{DISCUSSION}

For decades, lobectomy has remained the accepted standard of care for early-stage NSCLC (22). However, this standard is currently being challenged by SBRT, especially for the treatment of elderly patients and those with clinically significant comorbidities. Earlier reports had shown that SBRT can achieve comparable outcomes to surgical resection even in operable patients $(13,16,23,24)$. Two prospective phase II clinical trials (JCOG 0403 and RTOG 0618), assessing SBRT in operable stage I NSCLC, showed that the OS at 3 years was 76$85 \%$. This rate was similar to that reported for surgery $(14,15)$. However, most of these studies have been limited by different surgical approaches (thoracotomy and VATS), and mixed extents of resection (sublobar, lobectomy, bilobectomy, and pneumonectomy) (25). Fewer study focus on comparisons between SBRT and VATS (26). In addition, none of the four ongoing phase III trials comparing SBRT and surgical resection [VALOR (NCT02984761), POSTILV (NCT01753414), STABLE-MATES (NCT01622621), and RAXSIA (NCT03431415)] includes VATS. The relative effectiveness of treatments cannot be inferred in the absence of comparative data from modern minimally invasive techniques.

We performed a PSM pair analysis of outcomes of two potentially curative approaches for early-stage NSCLC. The PSM analysis identifies patients with similar characteristics, approximating the design of a randomized controlled trial. The results of this study indicated that the 3- and 5-year CSS rates associated with SBRT were comparable with those reported in patients who underwent VATS. Patients who received SBRT demonstrated promising LRC, despite undergoing only noninvasive staging of the lymph nodes. As expected, there were no significant differences observed between the two approaches in terms of LRC or DFS. The results reported in both groups were generally consistent with those currently available in the literature $(27,28)$. After 3 years, there appears to be a trend toward improved OS in patients who undergo surgery. We postulate that this finding was attributed to the poorer condition of patients who received SBRT versus that of patients who underwent VATS, despite the matching procedure. In addition, the long-term toxicity of SBRT should be monitored 
TABLE 1 | Characteristics of patients with early-stage NSCLC stratified according to treatment.

\begin{tabular}{|c|c|c|c|c|}
\hline \multirow[t]{2}{*}{ Variable } & \multirow{2}{*}{$\frac{\text { Overall cohort }}{n(\%)}$} & \multirow{2}{*}{$\frac{\text { SBRT }}{n(\%)}$} & \multirow{2}{*}{$\frac{\text { VATS lobectomy }}{n(\%)}$} & \multirow[t]{2}{*}{$p$} \\
\hline & & & & \\
\hline \multicolumn{5}{|c|}{ Sociodemographics } \\
\hline Age (years) & & & & $<0.001$ \\
\hline$<65$ & $320(56)$ & $19(17)$ & $301(66)$ & \\
\hline $65-74$ & $174(31)$ & $34(31)$ & $140(30)$ & \\
\hline$\geq 75$ & $73(13)$ & $56(52)$ & $17(4)$ & \\
\hline Gender & & & & $<0.001$ \\
\hline Male & $294(52)$ & 79 (73) & $215(47)$ & \\
\hline Female & $273(48)$ & $30(27)$ & $243(53)$ & \\
\hline FEV1\% predicted & & & & $<0.001$ \\
\hline$<30$ & $62(10)$ & $43(39)$ & $19(4)$ & \\
\hline $30-49$ & $101(19)$ & $24(22)$ & $77(17)$ & \\
\hline $50-79$ & $147(26)$ & $16(15)$ & $131(29)$ & \\
\hline$\geq 80$ & $257(45)$ & $26(24)$ & $231(50)$ & \\
\hline FEV1/FVC (\%) & & & & $<0.001$ \\
\hline$\geq 70$ & $547(96)$ & $91(83)$ & 456 (99) & \\
\hline$<70$ & $20(4)$ & $18(17)$ & $2(1)$ & \\
\hline KPS & & & & 0.033 \\
\hline$\geq 90$ & $513(90)$ & $89(82)$ & $424(92)$ & \\
\hline$<90$ & $54(10)$ & 20 (18) & $34(8)$ & \\
\hline $\mathrm{CCl}$ & & & & $<0.001$ \\
\hline 0 & $369(65)$ & $42(39)$ & $327(71)$ & \\
\hline $1-2$ & $170(30)$ & $58(53)$ & $112(25)$ & \\
\hline$\geq 3$ & $28(5)$ & $9(8)$ & $19(4)$ & \\
\hline \multicolumn{5}{|c|}{ Tumor characteristics } \\
\hline Tumor size (cm) & & & & 0.201 \\
\hline$\leq 2.0$ & $328(58)$ & $56(51)$ & $272(59)$ & \\
\hline $2.1-3.0$ & 187 (33) & $41(38)$ & $146(32)$ & \\
\hline $3.1-5.0$ & $52(9)$ & $12(11)$ & $40(9)$ & \\
\hline
\end{tabular}

SBRT, stereotactic body radiotherapy; NOS, not otherwise specified; NSCLC, non-small cell lung cancer; Ade, adenocarcinoma; SCC, squamous cell carcinoma; FEV1, forced expiratory volume in $1 \mathrm{~s}$; FEV1/FVC\%, FEV1 and forced vital capacity ratio; CCI, Charlson comorbidity index; KPS, Karnofsky performance status.

In bold: the difference was statistically significant.

TABLE 2 | Characteristics of propensity score-matched patients.

\begin{tabular}{|c|c|c|c|}
\hline \multirow[t]{2}{*}{ Variable } & \multirow{2}{*}{$\begin{array}{l}\text { SBRT } \\
\text { n (\%) }\end{array}$} & \multirow{2}{*}{$\frac{\text { VATS lobectomy }}{n(\%)}$} & \multirow[t]{2}{*}{$P$} \\
\hline & & & \\
\hline Age (years) & & & 0.455 \\
\hline Median (range) & 68 (47-83) & $67(45-83)$ & \\
\hline Gender & & & 1.000 \\
\hline Male & $31(60)$ & $31(60)$ & \\
\hline Female & $21(40)$ & $21(40)$ & \\
\hline FEV1\% predicted & & & 0.471 \\
\hline Median (range) & 79 (31-109) & $80(27-112)$ & \\
\hline FEV1/FVC (\%) & & & 0.391 \\
\hline Median (range) & $104(31-125)$ & 107 (54-119) & \\
\hline KPS & & & 0.918 \\
\hline Median (range) & $90(80-100)$ & $90(80-100)$ & \\
\hline $\mathrm{CCl}$ & & & 0.625 \\
\hline 0 & $30(58)$ & $36(69)$ & \\
\hline $1-2$ & $10(19)$ & $6(12)$ & \\
\hline$\geq 3$ & $12(23)$ & 10 (19) & \\
\hline Tumor size (cm) & & & 0.411 \\
\hline Median (range) & $2.0(0.5-5.0)$ & $2.0(0.8-4.0)$ & \\
\hline
\end{tabular}

SBRT, stereotactic body radiotherapy; VATS, video-assisted thoracoscopic surgery; FEV1, forced expiratory volume in 1 s; FEV1/FVC\%, FEV1 and forced vital capacity ratio; CCl, Charlson comorbidity index; KPS, Karnofsky performance status. to assess whether non-tumor deaths are caused by therapeutic factors. We hope our findings will increase the impetus to expand the use of SBRT and to conduct high-quality clinical effectiveness trials.

It's worth noting that our outcomes contradict to some of the recent suggestion that lobectomy is superior to SBRT. In some retrospective studies, patients selected for VATS have improved survival compared with SBRT (29-33). In these studies, routine systematic mediastinal lymph node dissection identified candidates for adjuvant chemotherapy, which may be associated with a significant difference in OS and CSS. In addition, the SBRT group consisted mainly of medically inoperable patients with poor pulmonary function and severe comorbidities, which accounts for their relatively low rates of survival. In D. Detillon's study, the elderly patients with stage I NSCLC undergoing VATS lobectomy have a better OS than patients undergoing SBRT. However, due to the characters of the database, in their study, the cause of death was unknown. In addition, performance status and pulmonary function were not available in most of patients, which are very important for guiding treatment choice and prognosis (25). Further robust and long follow-up studies are warranted to demonstrate that SBRT may achieve comparable results with those reported after surgery.

There was no histopathologic proof of malignancy obtained in $22 \%$ of the patients who received radiotherapy. The probability of malignancy in 11 SBRT patients without a pretreatment pathological diagnosis was calculated using a combination of clinical, radiological, and PET/CT findings, as previously described. The national radiotherapy guidelines in the Netherlands indicate that patients without histologic confirmation undergo radiotherapy in case of: (a) a new or growing lesion shown on CT scans with characteristics of malignancy; (b) a high risk for developing lung cancer based on age and smoking history; and (c) PET/CT-positive lesions (17). The probability of benign disease in these patients is merely $4.3 \%$ (34). However, it remains our policy to obtain a pretreatment diagnosis in all patients, if possible.

An academic radiologist reviewed the post-SBRT imaging findings to score patterns of failure in this study. The majority of SBRT-induced lung injuries can result in a CT density change, which occasionally mimics tumor recurrence. This renders the distinction between recurrence and radiation fibrosis during follow-up challenging. In addition, it is difficult to differentiate between intrapulmonary metastases and secondary primary lung carcinoma. Therefore, it is important to identify and validate high-risk radiological features appearing on serial CT scans that suggest recurrence (i.e., enlarging opacity, continuous enlargement, enlargement after 12 months, bulging margin, linear margin disappearance, and loss of air bronchogram). The presence of high-risk radiological features coupled with a $\mathrm{PET} / \mathrm{CT}$ maximum standardized uptake value $>5$ are highly suggestive of tumor recurrence $(35,36)$.

Toxicity is particularly important when considering options for the treatment of cancer with similar long-term survival. In this study, we observed limited toxicity in both groups. One patient developed a grade 3 complication (radioactive pneumonitis), and 

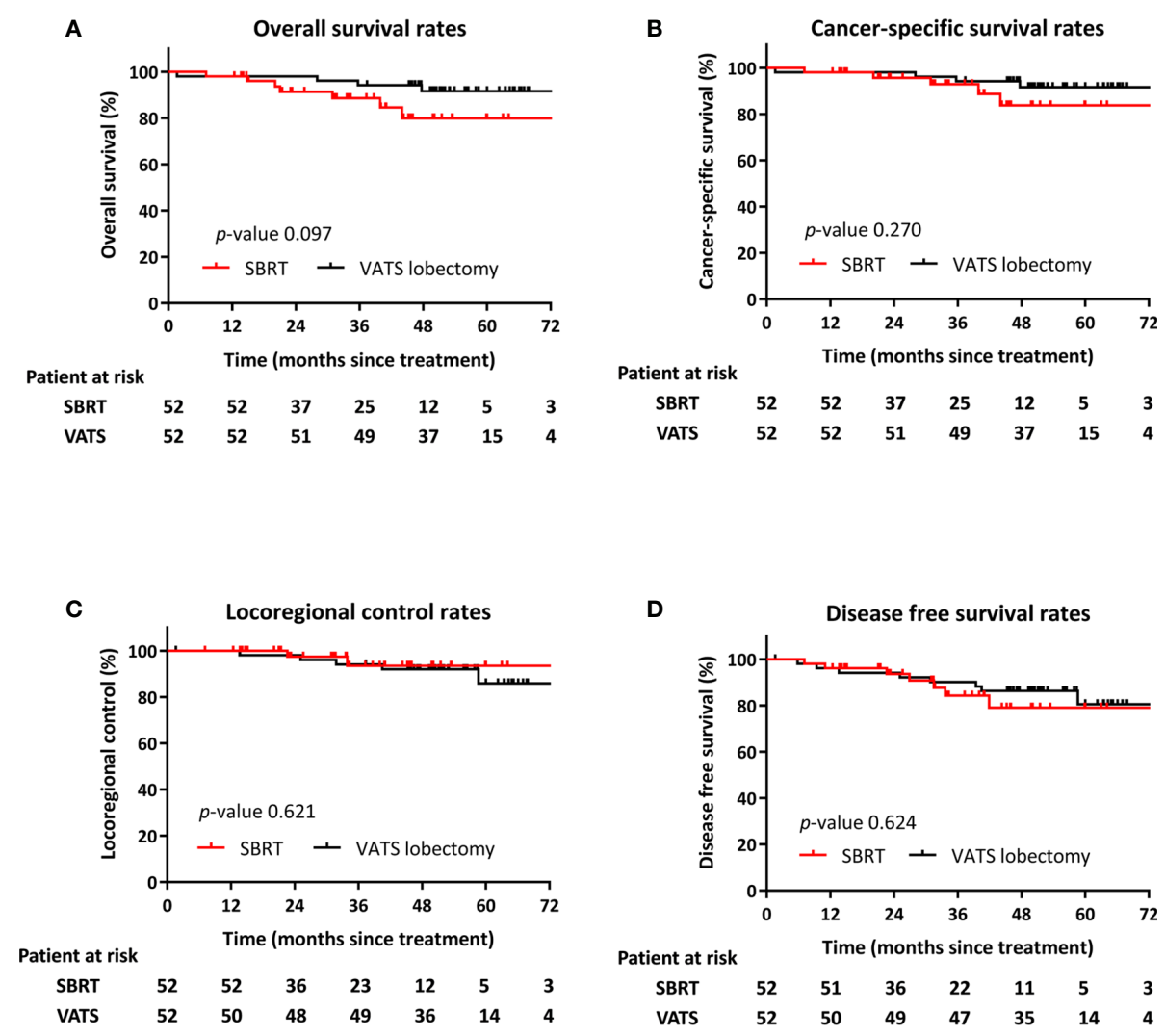

FIGURE 2 | Kaplan-Meier curves following propensity score-matching for overall survival (A), cancer-specific survival (B), locoregional control (C), and progressionfree survival (D) of patients after VATS lobectomy or SBRT.

TABLE 3 | Complications after surgery and SBRT.

\begin{tabular}{lcccc}
\hline Toxicity/Complication & No. (\%) & Grade 1 & Grade 2 & Grade 3 \\
\hline VATS lobectomy $(\mathrm{n}=52)$ & & & & \\
Pain & $5(10)$ & 5 & 0 & 0 \\
Cough & $19(37)$ & 10 & 9 & 0 \\
Shortness of breath & $22(42)$ & 14 & 8 & 0 \\
Hoarseness & $3(6)$ & 3 & 0 & 0 \\
Pneumonia & $10(19)$ & 5 & 1 & 4 \\
Pleural effusion & $13(25)$ & 8 & 5 & 0 \\
SBRT (n = 52) & & & & \\
Radiation pneumonitis & $18(35)$ & 14 & 3 & 1 \\
Chest pain & $3(6)$ & 3 & 0 & 0 \\
Cough & $26(50)$ & 19 & 7 & 0 \\
Rib fracture & $0(0)$ & 0 & 0 & 0 \\
Shortness of breath & $12(23)$ & 10 & 2 & 0 \\
Hoarseness & $2(4)$ & 2 & 0 & 0 \\
\hline
\end{tabular}

SBRT, stereotactic body radiotherapy; VATS, video-assisted thoracoscopic surgery.

there were no deaths attributable to SBRT. In the surgery group, the incidence of supraventricular arrhythmias and empyema was lower, and grade 3 complications were observed in four patients. Of note, one patient (2\%) expired due to perioperative infection within 30 days after surgery. The role of SBRT as a curative modality for early-stage NSCLC may become a more attractive option, considering the comparable overall clinical outcomes and low rates of treatment-related morbidity and mortality versus surgery.

A strength of our analysis is that the demographic- and tumormatching factors at baseline were relatively comprehensive with limited variability. The strict 0.1 maximum caliper width for propensity score difference guaranteed an accurate PSM. Furthermore, the patient population truly reflected clinical practice, rather than being composed of selected, relatively suitable patients, which is often the case in clinical trials.

The limitations of this study should be acknowledged. Firstly, although the cohorts were accurately matched, this remains a retrospective study. Therefore, unidentified or unrecorded factors (e.g., histology and grade) may have played a role in selected patients. Squamous cell carcinoma and low-grade differentiation have been found associated with worse LRC and OS $(37,38)$. Secondly, patients receiving SBRT were clinically staged, while patients undergoing VATS were ultimately pathologically staged. Most SBRT patients did not undergo nodal staging or dissection in our analysis. Thus, it is likely that these patients were underestimated in this study, contributing to a pathological staging bias in favor of surgery. In addition, given the relatively small sample size and limited follow-up of our cohort, there is limited precision in estimating differences in outcomes across treatment 
groups. Finally, the optimal identification of acute and late treatment-related adverse events is another weakness of this study.

With the emergence of new technologies for the treatment of lung carcinoma (i.e., SBRT), and widespread introduction of lung cancer screening, thoracic surgeons will be faced with smaller lung lesions that may be amenable to these alternative treatments. The combination of these factors with the aging population characterized by more comorbidities indicates that thoracoscopic resection techniques present a favorable, more palatable option for many patients versus open procedures. Currently, there are insufficient data to assist clinicians and patients in reaching a decision regarding the optimal treatment for early-stage NSCLC. Retrospective reviews, may provide clues regarding the most appropriate management. The present findings provided useful information in answering these and other unresolved questions regarding SBRT.

\section{CONCLUSIONS}

The results of this propensity score-matching analysis demonstrated that SBRT achieved comparable overall clinical outcomes to those observed with VATS. These data may provide a decision-making reference between healthcare providers and patients. Randomized trials investigating a number of minimally invasive techniques are required to accurately compare outcomes between these approaches.

\section{REFERENCES}

1. Kang HR, Cho JY, Lee SH, Lee YJ, Park JS, Cho YJ, et al. Role of Low-Dose Computerized Tomography in Lung Cancer Screening among NeverSmokers. J Thorac Oncol (2019) 14(3):436-44. doi: 10.1016/j.jtho.2018.11.002

2. Aberle DR, DeMello S, Berg CD, Black WC, Brewer B, Church TR, et al. Results of the two incidence screenings in the National Lung Screening Trial. N Engl J Med (2013) 369(10):920-31. doi: 10.1056/NEJMoa 1208962

3. Howington JA, Blum MG, Chang AC, Balekian AA, Murthy SC. Treatment of stage I and II non-small cell lung cancer: Diagnosis and management of lung cancer, 3rd ed: American College of Chest Physicians evidence-based clinical practice guidelines. Chest (2013) 143(5 Suppl):e278S-313S. doi: 10.1378/ chest.12-2359

4. Darling GE, Allen MS, Decker PA, Ballman K, Malthaner RA, Inculet RII, et al. Number of lymph nodes harvested from a mediastinal lymphadenectomy: results of the randomized, prospective American College of Surgeons Oncology Group Z0030 trial. Chest (2011) 139 (5):1124-9. doi: 10.1378/chest.10-0859

5. Nwogu CE, D'Cunha J, Pang H, Gu L, Wang X, Richards WG, et al. VATS lobectomy has better perioperative outcomes than open lobectomy: CALGB 31001, an ancillary analysis of CALGB 140202 (Alliance). Ann Thorac Surg (2015) 99(2):399-405. doi: 10.1016/j.athoracsur.2014.09.018

6. Burt BM, Kosinski AS, Shrager JB, Onaitis MW, Weigel T. Thoracoscopic lobectomy is associated with acceptable morbidity and mortality in patients with predicted postoperative forced expiratory volume in 1 second or diffusing capacity for carbon monoxide less than $40 \%$ of normal. J Thorac Cardiovasc Surg (2014) 148(1):19-28, dicussion 28-29.e1. doi: 10.1016/ j.jtcvs.2014.03.007

7. Paul S, Sedrakyan A, Chiu YL, Nasar A, Port JL, Lee PC, et al. Outcomes after lobectomy using thoracoscopy vs thoracotomy: a comparative effectiveness

\section{DATA AVAILABILITY STATEMENT}

The original contributions presented in the study are included in the article/supplementary material. Further inquiries can be directed to the corresponding authors.

\section{ETHICS STATEMENT}

Ethical review and approval was not required for the study on human participants, in accordance with the local legislation and institutional requirements.

\section{AUTHOR CONTRIBUTIONS}

$\mathrm{BD}$ was responsible for project conceptualization, data collection and analysis, data interpretation, writing of the manuscript, and all manuscript revisions. XZ was responsible for project conceptualization, manuscript revisions, and editing of the manuscript. ZS was responsible for statistical analysis and editing of the manuscript. YJ was responsible for SBRT patient data collection and editing of the manuscript. FL was responsible for score the imaging results of follow-up patients. JW and MC were responsible for project conceptualization and editing of the manuscript and should be considered the guarantor for the article as a whole. All authors contributed to the article and approved the submitted version.

analysis utilizing the Nationwide Inpatient Sample database. Eur J Cardiothorac Surg (2013) 43(4):813-7. doi: 10.1093/ejcts/ezs428

8. Detillon D, Veen EJ. Postoperative Outcome After Pulmonary Surgery for Non-Small Cell Lung Cancer in Elderly Patients. Ann Thorac Surg (2018) 105 (1):287-93. doi: 10.1016/j.athoracsur.2017.07.032

9. Detillon D, Driessen EJM, Aarts MJ, Janssen-Heijnen MLG, van Eijck CHJ, Veen EJ. Changes in treatment patterns and survival in elderly patients with stage I non-small-cell lung cancer with the introduction of stereotactic body radiotherapy and video-assisted thoracic surgery. Eur J Cancer (2018) 101:307. doi: 10.1016/j.ejca.2018.06.016

10. Tandberg DJ, Tong BC, Ackerson BG, Kelsey CR. Surgery versus stereotactic body radiation therapy for stage I non-small cell lung cancer: A comprehensive review. Cancer (2018) 124(4):667-78. doi: 10.1002/cncr.31196

11. Vansteenkiste J, Crino L, Dooms C, Douillard JY, Faivre-Finn C, Lim E, et al. 2nd ESMO Consensus Conference on Lung Cancer: early-stage non-small-cell lung cancer consensus on diagnosis, treatment and follow-up. Ann Oncol (2014) 25(8):1462-74. doi: 10.1093/annonc/mdu089

12. Grills IS, Mangona VS, Welsh R, Chmielewski G, McInerney E, Martin S, et al. Outcomes after stereotactic lung radiotherapy or wedge resection for stage I non-small-cell lung cancer. J Clin Oncol (2010) 28(6):928-35. doi: 10.1200/ jco.2009.25.0928

13. Onishi $H$, Shirato $H$, Nagata $Y$, Hiraoka $M$, Fujino $M$, Gomi $K$, et al. Stereotactic body radiotherapy (SBRT) for operable stage I non-small-cell lung cancer: can SBRT be comparable to surgery? Int J Radiat Oncol Biol Phys (2011) 81(5):1352-8. doi: 10.1016/j.ijrobp.2009.07.1751

14. Timmerman RD, Paulus R, Pass HII, Gore EM, Edelman MJ, Galvin J, et al. Stereotactic Body Radiation Therapy for Operable Early-Stage Lung Cancer: Findings From the NRG Oncology RTOG 0618 Trial. JAMA Oncol (2018) 4 (9):1263-6. doi: 10.1001/jamaoncol.2018.1251

15. Nagata Y, Hiraoka M, Shibata T, Onishi H, Kokubo M, Karasawa K, et al. Prospective Trial of Stereotactic Body Radiation Therapy for Both Operable 
and Inoperable T1N0M0 Non-Small Cell Lung Cancer: Japan Clinical Oncology Group Study JCOG0403. Int J Radiat Oncol Biol Phys (2015) 93 (5):989-96. doi: 10.1016/j.ijrobp.2015.07.2278

16. Chang JY, Senan S, Paul MA, Mehran RJ, Louie AV, Balter P, et al. Stereotactic ablative radiotherapy versus lobectomy for operable stage I non-small-cell lung cancer: a pooled analysis of two randomised trials. Lancet Oncol (2015) 16(6):630-7. doi: 10.1016/s1470-2045(15)70168-3

17. Lagerwaard FJ, Verstegen NE, Haasbeek CJ, Slotman BJ, Paul MA, Smit EF, et al. Outcomes of stereotactic ablative radiotherapy in patients with potentially operable stage I non-small cell lung cancer. Int J Radiat Oncol Biol Phys (2012) 83(1):348-53. doi: 10.1016/j.ijrobp.2011.06.2003

18. Dong B, Wang J, Xu Y, Hu X, Shao K, Li J, et al. Comparison of the Efficacy of Stereotactic Body Radiotherapy versus Surgical Treatment for Early-Stage Non-Small Cell Lung Cancer after Propensity Score Matching. Transl Oncol (2019) 12(8):1032-7. doi: 10.1016/j.tranon.2019.04.015

19. Chen G, Dong B, Shan G, Zhang X, Tang H, Li Y, et al. Choice of immobilization of stereotactic body radiotherapy in lung tumor patient by BMI. BMC Cancer (2019) 19(1):583. doi: 10.1186/s12885-019-5767-1

20. Dong B, Wang J, Zhu X, Chen Y, Xu Y, Shao K, et al. Comparison of the outcomes of stereotactic body radiotherapy versus surgical treatment for elderly $(>/=70)$ patients with early-stage non-small cell lung cancer after propensity score matching. Radiat Oncol (2019) 14(1):195. doi: 10.1186/ s13014-019-1399-5

21. Timmerman R, Paulus R, Galvin J, Michalski J, Straube W, Bradley J, et al. Stereotactic body radiation therapy for inoperable early stage lung cancer. Jama (2010) 303(11):1070-6. doi: 10.1001/jama.2010.261

22. Ettinger DS, Wood DE, Akerley W, Bazhenova LA, Borghaei H, Camidge DR, et al. NCCN Guidelines Insights: Non-Small Cell Lung Cancer, Version 4.2016. J Natl Compr Canc Netw (2016) 14(3):255-64. doi: 10.6004/jnccn.2016.0031

23. Palma DA, Senan S. Improving outcomes for high-risk patients with earlystage non-small-cell lung cancer: insights from population-based data and the role of stereotactic ablative radiotherapy. Clin Lung Cancer (2013) 14(1):1-5. doi: 10.1016/j.cllc.2012.06.005

24. Louie AV, Rodrigues G, Hannouf M, Zaric GS, Palma DA, Cao JQ, et al. Stereotactic body radiotherapy versus surgery for medically operable Stage I non-small-cell lung cancer: a Markov model-based decision analysis. Int $\mathrm{J}$ Radiat Oncol Biol Phys (2011) 81(4):964-73. doi: 10.1016/j.ijrobp.2010.06.040

25. Detillon D, Aarts MJ, De Jaeger K, Van Eijck CHJ, Veen EJ. Video-assisted thoracic lobectomy versus stereotactic body radiotherapy for stage I nonsmall cell lung cancer in elderly patients: a propensity matched comparative analysis. Eur Respir J (2019) 53(6):1801561. doi: 10.1183/13993003.01561-2018

26. Boada M, Guzman R, Montesinos M, Libreros A, Guirao A, Sanchez-Lorente D, et al. Upstaging, Centrality And Survival In Early Stage Non-Small Cell Lung Cancer Video-Assisted Surgery. Lung Cancer (2019) 134:254-8. doi: 10.1016/j.lungcan.2019.06.030

27. Verstegen NE, Oosterhuis JW, Palma DA, Rodrigues G, Lagerwaard FJ, van der Elst A, et al. Stage I-II non-small-cell lung cancer treated using either stereotactic ablative radiotherapy (SABR) or lobectomy by video-assisted thoracoscopic surgery (VATS): outcomes of a propensity score-matched analysis. Ann Oncol (2013) 24(6):1543-8. doi: 10.1093/annonc/mdt026

28. Paul S, Isaacs AJ, Treasure T, Altorki NK, Sedrakyan A. Long term survival with thoracoscopic versus open lobectomy: propensity matched comparative analysis using SEER-Medicare database. Bmj (2014) 349:g5575. doi: 10.1136/bmj.g5575
29. Puri V, Crabtree TD, Bell JM, Broderick SR, Morgensztern D, Colditz GA et al. Treatment Outcomes in Stage I Lung Cancer: A Comparison of Surgery and Stereotactic Body Radiation Therapy. J Thorac Oncol (2015) 10(12):177684. doi: $10.1097 /$ jto. 0000000000000680

30. Kidane B. Stereotactic body radiation therapy versus video-assisted thoracoscopic surgery in stage I lung cancer: Honesty in the face of uncertainty. J Thorac Cardiovasc Surg (2018) 155(1):365-6. doi: 10.1016/ j.jtcvs.2017.08.059

31. Cornwell LD, Echeverria AE, Samuelian J, Mayor J, Casal RF, Bakaeen FG, et al. Video-assisted thoracoscopic lobectomy is associated with greater recurrence-free survival than stereotactic body radiotherapy for clinical stage I lung cancer. J Thorac Cardiovasc Surg (2018) 155(1):359-402. doi: 10.1016/j.jtcvs.2017.07.065v

32. Hamaji M, Chen F, Matsuo Y, Kawaguchi A, Morita S, Ueki N, et al. Videoassisted thoracoscopic lobectomy versus stereotactic radiotherapy for stage I lung cancer. Ann Thorac Surg (2015) 99(4):1122-9. doi: 10.1016/j.athoracsur. 2014.11.009

33. Rosen JE, Salazar MC, Wang Z, Yu JB, Decker RH, Kim AW, et al. Lobectomy versus stereotactic body radiotherapy in healthy patients with stage I lung cancer. J Thorac Cardiovasc Surg (2016) 152(1):44-54.e9. doi: 10.1016/j.jtcvs. 2016.03.060

34. Belgers EH, Siebenga J, Bosch AM, van Haren EH, Bollen EC. Complete video-assisted thoracoscopic surgery lobectomy and its learning curve. A single center study introducing the technique in The Netherlands. Interact Cardiovasc Thorac Surg (2010) 10(2):176-80. doi: 10.1510/icvts.2009.212878

35. Huang K, Senthi S, Palma DA, Spoelstra FO, Warner A, Slotman BJ, et al. High-risk CT features for detection of local recurrence after stereotactic ablative radiotherapy for lung cancer. Radiother Oncol (2013) 109(1):51-7. doi: 10.1016/j.radonc.2013.06.047

36. Huang K, Dahele M, Senan S, Guckenberger M, Rodrigues GB, Ward A, et al. Radiographic changes after lung stereotactic ablative radiotherapy (SABR)can we distinguish recurrence from fibrosis? A systematic review of the literature. Radiother Oncol (2012) 102(3):335-42. doi: 10.1016/ j.radonc.2011.12.018

37. Woody NM, Stephans KL, Andrews M, Zhuang T, Gopal P, Xia P, et al. A Histologic Basis for the Efficacy of SBRT to the lung. J Thorac Oncol (2017) 12 (3):510-9. doi: 10.1016/j.jtho.2016.11.002

38. Leeman JE, Rimner A, Montecalvo J, Hsu M, Zhang Z, von Reibnitz D, et al. Histologic Subtype in Core Lung Biopsies of Early-Stage Lung Adenocarcinoma is a Prognostic Factor for Treatment Response and Failure Patterns After Stereotactic Body Radiation Therapy. Int J Radiat Oncol Biol Phys (2017) 97(1):138-45. doi: 10.1016/j.ijrobp.2016.09.037

Conflict of Interest: The authors declare that the research was conducted in the absence of any commercial or financial relationships that could be construed as a potential conflict of interest.

Copyright $\odot 2020$ Dong, Zhu, Shu, Ji, Lu, Wang and Chen. This is an open-access article distributed under the terms of the Creative Commons Attribution License (CC BY). The use, distribution or reproduction in other forums is permitted, provided the original author(s) and the copyright owner(s) are credited and that the original publication in this journal is cited, in accordance with accepted academic practice. No use, distribution or reproduction is permitted which does not comply with these terms. 